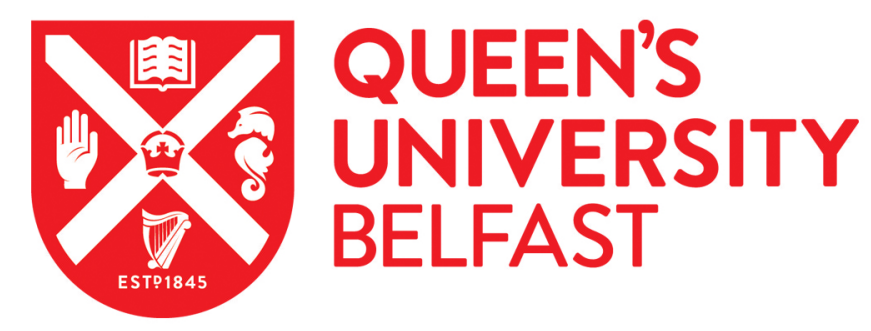

\title{
Managing Patients at Risk of Medication Related Complications Requiring Dental Extractions in Primary Care
}

Toole, J., McKenna, G., \& Smyth, J. (2020). Managing Patients at Risk of Medication Related Complications Requiring Dental Extractions in Primary Care. Primary Dental Journal, 9(3), 54-58.

https://doi.org/10.1177/2050168420943977

Published in:

Primary Dental Journal

Document Version:

Peer reviewed version

Queen's University Belfast - Research Portal:

Link to publication record in Queen's University Belfast Research Portal

Publisher rights

(C) 2020 The Authors.

This work is made available online in accordance with the publisher's policies. Please refer to any applicable terms of use of the publisher.

\section{General rights}

Copyright for the publications made accessible via the Queen's University Belfast Research Portal is retained by the author(s) and / or other copyright owners and it is a condition of accessing these publications that users recognise and abide by the legal requirements associated with these rights.

Take down policy

The Research Portal is Queen's institutional repository that provides access to Queen's research output. Every effort has been made to ensure that content in the Research Portal does not infringe any person's rights, or applicable UK laws. If you discover content in the Research Portal that you believe breaches copyright or violates any law, please contact openaccess@qub.ac.uk. 
Managing patients at risk of medication related complications requiring dental extractions in primary care.

\begin{abstract}
When undertaking dental extractions in modern dental practice, two of the complications that have the potential to cause most apprehension for clinicians are the risks of osteonecrosis of the jaws and uncontrollable haemorrhage. This is especially the case when treating older patients because of the increased likelihood of co-morbidities and accompanying polypharmacy which can predispose patients to these problems. Specific medications of concern to practitioners in relation to osteonecrosis risk are antiangiogenic and anti resorptive drugs. Patients taking dual antiplatelet therapy and direct oral anticoagulants require consideration in relation to bleeding risk. With these medications coming increasingly to the fore over recent years guidance has been developed by organisations such as the Scottish Dental Clinical Effectiveness Programme (SDCEP). Appropriate use of these guideline should ensure that patients felt to be at particular risk of these complications can frequently be safely managed in primary care. This article aims to provide advice on recognising patients at risk, and to discuss how to utilise key messages within published guidelines when making treatment decisions. The overall intent is to help primary care clinicians who are likely to encounter these patients more and more.
\end{abstract}

Keywords: Extractions, osteonecrosis, antiplatelet, anticoagulant

Learning outcomes

By the end of this article you should be able to:

- Recognise patients at risk of medication related osteonecrosis of the jaw, and apply key points from published guidelines when formulating treatment plans

- Recognise patients at risk of post-operative haemorrhage due to dual antiplatelet and direct oral anticoagulant therapies, and apply key points from published guidelines when formulating treatment plans

Authors

Jamie Toole, Consultant / Honorary Clinical Lecturer in Oral Surgery, Centre for Dentistry, Queen's University Belfast

Joanna Smyth, Clinical Teaching Fellow in Oral Surgery, Centre for Dentistry, Queen's University Belfast

Gerry McKenna, Senior Lecturer / Consultant in Restorative Dentistry, Centre for Public Health, Queen's University Belfast 


\section{Introduction}

Within the context of an aging population, increasing numbers of elderly patients are attending general dental practice for routine dental care. It is perhaps no surprise that these patients can provide challenges for the clinician due to a range of systemic co-morbidities and accompanying polypharmacy. The most recent United Kingdom (UK) Adult Dental Health Survey reveals that approximately $80 \%$ of patients aged 65 or over are partially dentate (1). This is in stark contrast to findings three decades previously, at which stage only $20 \%$ of this group had any retained natural teeth (1). Given this epidemiological shift there is an increasingly need for dentists in primary care to carry out oral surgery procedures for older patients, including extractions. It follows that this patient group needs to be managed appropriately both from a dental and medical viewpoint. Two of the most significant concerns for clinicians undertaking dental extractions are the risk of osteonecrosis of the jaws and uncontrollable haemorrhage. The risk of each of these can be increased by co-morbidities commonly afflicting elderly patients, and the medications used to treat them. This article will discuss the most common challenges of managing older patients in primary care and appropriate clinical decision making for this patient group.

\section{Medication-related Osteonecrosis of the Jaw}

Medication-related osteonecrosis of the jaw (MRONJ) is defined as exposed bone or bone that can be probed in the maxillofacial region, that has persisted for more than 8 weeks in patients who are currently or have previously been treated with antiresorptive or antiangiogenic drugs, and who have no history of radiotherapy to the jaws or obvious metastatic disease of the jaws (2). Dental extractions have been shown to be the initiating factor for $73 \%$ of cases of MRONJ(6). If the number of dental extractions required in this patient cohort was reduced, it is likely that there could be a significant drop in the incidence of MRONJ along with a reduction in the morbidity suffered by these patients.

\section{Patients at risk}

Antiangiogenic drugs are used in the treatment of various cancers with the aim of preventing new blood vessel formation. Antiresorptive drugs can also be involved in cancer treatment but are more frequently used to treat osteoporosis. Osteoporosis affects a significant number of patients in the UK with $21.8 \%$ of females over the age of 50 and $6.8 \%$ of males being diagnosed with this condition (3). In 2017 there were over 500000 fragility fractures in the UK, resulting in the loss of 200000 quality-adjusted life years (3). Antiresorptive drugs have been shown to reduce the risk of fragility fractures and the associated morbidity and mortality $(4,5)$. Commonly prescribed antiresorptive drugs include bisphosphonates and Denosumab, a human monoclonal antibody. Whilst these treatments are systemically beneficial, the risk of MRONJ needs to be considered both by the patient and the treating clinician when undertaking invasive procedures such as extractions.

MRONJ is classified as a rare complication with the incidence in the UK reported as between 1 in 1262-4419 cases per year in the population (6). However, the risk for patients receiving oral bisphosphonates for osteoporosis and who require extractions has been shown to increase to as high as 1 in 296 cases (7). 


\section{Clinical decision-making based on key points from published guidelines}

The Scottish Dental Clinical Effectiveness Programme (SDCEP) have produced guidelines for managing patients who are at risk of MRONJ that have also been accepted by the National Institute of Clinical Excellence (NICE) (8,9). Alongside the full guidance, a 'Guidance in Brief' document is available, which provides a useful quick reference tool (10). With the backing of NICE and the acceptance of these guidelines by the Department of Health in Northern Ireland, they can be considered as UK-wide guidance. The guidelines categorise the risk of a patient developing MRONJ into low or high risk. Patients considered to be at low risk are those that:

- Are being treated for osteoporosis or other non-malignant disease of the bone with oral bisphosphonates for less than 5 years and who are not concurrently taking systemic glucocorticoids

- Are being treated for osteoporosis or other non-malignant disease of the bone with quarterly or yearly intravenous infusions of bisphosphonates for less than 5 years and who are not concurrently taking systemic glucocorticoids

- Are being treated for osteoporosis or other non-malignant disease of the bone with Denosumab and who are not concurrently taking systemic glucocorticoids (9)

Although there is no clear clinical evidence of the significance of concurrent treatment with systemic glucocorticoids, the importance can be inferred through in vitro research on the effects of bisphosphonates on the immune system. Several studies have found that bisphosphonates affect macrophage viability $(11,12)$. The American Association of Oral and Maxillofacial Surgeons (AAOMS) have produced a position paper that expands this theory to include the role of any innate or acquired immune dysfunction as risk factors for MRONJ which would seem like a logical extension (13).

The definition of 5 years of oral bisphosphonate treatment to categorize low risk patients is also somewhat controversial because of the lack of available research. In contrast AAOMS uses 4 years of bisphosphonate treatment to differentiate risk levels (13). A UK wide survey found the median number of years of bisphosphonate treatment before onset of MRONJ to be 4 years (6). The fact that increasing duration of treatment with bisphosphonates also increases the risk of MRONJ is beyond doubt, but an exact calculation is impossible. Clinicians should keep this at the forefront of their minds when discussing treatment options with patients. Denosumab is not thought to have the same cumulative risk as bisphosphonates due to differing pharmacology. Denosumab acts directly on the RANK ligand and is not incorporated into a patient's skeleton unlike bisphosphonates (14).

According to the SDCEP guidelines patients are considered to be at high risk of developing MRONJ if they have:

- Been treated for osteoporosis or other non-malignant disease of the bone with bisphosphonates or Denosumab for any length of time and who are concurrently taking systemic glucocorticoids

- Been treated for osteoporosis or other non-malignant disease of the bone with oral or infused bisphosphonates for greater than 5 years

- Been treated with antiresorptive or antiangiogenic medications as part of their treatment for cancer

- Been previously diagnosed with MRONJ(9) 
It is extremely important to remember that patients who are no longer taking bisphosphonates or Denosumab are still at risk of developing MRONJ (9). Due to the binding of bisphosphonates into the skeleton patients can be at risk for many years after stopping bisphosphonate treatment.

Denosumab is administered subcutaneously every 6 months as part of osteoporosis treatment and is therefore often not noted as part of patients' regular prescription medications. It is therefore essential to ask patients specific questions regarding these drugs during the medical history, especially if they reveal a diagnosis of osteoporosis.

It is widely agreed that for patients who are planned for treatment with antiresorptive drugs it is important to ensure that they are dentally fit prior to undertaking treatment. This means providing them with routine operative dental care and removing any teeth with a poor prognosis. The importance of diet and oral hygiene advice cannot be underestimated as part of a broad preventative message. It can be useful to discuss the risk of MRONJ with patients, which will hopefully support information they have already received from the healthcare professional prescribing the antiresorptive treatment. There can be a difficult balance to strike during this conversation as the patients need to understand the risk so they are encouraged to maintain their dentition, whilst not discouraged from taking a medication which will reduce their risk of fractures.

Patients who are at a high risk of MRONJ should be provided with normal dental care but should only have extractions when all other reasonable treatments have been considered. Dental infection can initiate MRONJ, accounting for $5 \%$ of cases, so patients are at risk if teeth that are a potential source of infection are left untreated (6). There is no requirement to restore a tooth to function to remove it as a potential source of infection. Undertaking root canal treatment and doming over roots can remove them as a potential source of infection, and provides no significant risk of MRONJ. These treatment plans can take much longer than one containing multiple extractions, but considering that only $25 \%$ of patients who get MRONJ are likely to heal (even with treatment) it is well worth the effort (6). The most likely outcome for a patient who develops MRONJ is that their condition is stabilised, but some will develop a progressive form of the disease(6). Should extractions be the only option for a patient deemed to be at high risk of developing MRONJ, it is essential to discuss the risks with them fully beforehand. If extractions are undertaken, patients must be reviewed and referred appropriately if you suspect MRONJ (9).

\section{Key points}

The key to managing extractions in older patients at risk of developing MRONJ is to ensure:

- An accurate risk assessment is undertaken prior to undertaking treatment with particular attention devoted to the medical history, including prescribed medications

- A thorough discussion about the treatment options is undertaken with the patient to ensure that they aware of their risk and to enable them to make an informed treatment decision

- Extractions are avoided for high risk patients unless absolutely necessary whilst still ensuring removal of any source of infection (for example, root canal treatment and doming over of retained roots) 


\section{Uncontrolled haemorrhage}

Thromboembolic disease constitutes a leading cause of mortality and morbidity worldwide.

Anticoagulants and antiplatelet drugs are an essential tool in the management of patients with thromboembolic disease due to their role in preventing the blood hypercoagulability which plays a central role in thrombogenesis. These drugs are commonly prescribed for older adults due to the chronic nature of thromboembolic disease. Dental extractions by their nature will induce bleeding and this may be considerable if more invasive procedures are required such as alveolar bone removal to facilitate surgical removal of teeth.

In recent years there have been a growing number of antiplatelet drugs used to prevent cardiovascular events. Patients undergoing percutaneous coronary interventions (e.g. placement of stents) are routinely started on dual antiplatelet therapy for between 6 and 12 months postoperatively, increasing to 36 months depending on the response (15). In the past, these drugs were used for much shorter periods of time. Since, in general, invasive dental treatment is best avoided in the short-term following cardiac procedures, dentists rarely saw patients on dual antiplatelet therapy. In recent years the increased treatment times mean that it is now much more common to see patients on these medication combinations. As they are designed to prevent clotting, they clearly cause some anxiety for dentists undertaking surgical procedures.

\section{Clinical decision-making based on key points from published guidelines}

The concern around uncontrollable haemorrhage is partly supported by meta-analyses of extractions in patients on dual antiplatelet therapy that found that, rather unsurprisingly, these patients are at an increased risk of bleeding compared to patients who are not taking antiplatelet medications $(16,17)$. However, other studies have not found these differences to be statistically significant (1820). The percentages of patients suffering post-operative bleeding events was relatively low at between 4.2 and $8.3 \%$ for dual antiplatelet therapy compared to control groups $(18,20,21)$. The most important aspect of these studies clinically is that all of the bleeding events were minor and controlled by local measures(16, 18-21). The authors drew the conclusion that dual antiplatelet therapy should not be interrupted for dental extractions $(16,18-21)$. As with MRONJ, the SDCEP have also produced guidance for patients taking anticoagulants and antiplatelet drugs (22) and there is also a 'Quick Reference Guide' to accompany the full guidance (23). These guidelines support this conclusion (22). However, it is suggested that clinicians should contemplate limiting the area being treated, stage treatment if required and consider packing and suturing the sockets when undertaking extractions (22). As with MRONJ it is important to discuss the risk with patients preoperatively and provide them with the necessary post-operative advice about what to do should they suffer problems with bleeding.

Newer generations of anticoagulants have also increased in use within the past few years. Originally termed Novel Oral Anticoagulants (NOACs) they are now frequently referred to as Direct Oral Anticoagulants (DOACs). These include factor Xa inhibitors (Rivaroxaban, Apixaban and Edoxaban) as well as direct thrombin inhibitors (Dabigatran). The SDCEP guidelines also cover these medications(22). In addition to considering the risk of a patient bleeding based on their medical conditions, such as chronic kidney disease, liver disease or haematological disease, and any medications they may take, the guidelines also suggest procedures that have a low or high risk of post-operative haemorrhage (22). The low risk group includes procedures such as simple extractions of 1 to 3 teeth, incision and drainage of intra-oral swellings, subgingival scaling and direct 
restorations (22). Procedures considered to have a higher risk of post-operative bleeding include complex extractions (defined as extractions which may be likely to have surgical complications), flap raising procedures, gingival recontouring and biopsies (22).

For patients who require a procedure with a low risk of post-operative bleeding the SDCEP guidelines advise that patients should not discontinue or delay taking their DOAC (22). Again the recommendations are to limit the initial treatment area and use local measures such as packing and suturing (22). It is also advised that patients should be treated in the morning (22). Whilst this has the disadvantage of the extraction being more likely to coincide with a peak of drug concentration assuming that the patient has taken a morning dose, it does make it easier to manage any complications during normal working hours (22). When these guidelines were originally published there was very little evidence available on this subject, however several studies have subsequently shown this to be a safe way of managing patients on DOACs (24-26).

For patients who are undergoing procedures that carry a higher risk of post-operative bleeding, the SDCEP guidelines recommend delaying the morning dose of the DOAC if patients take it once a day (Rivaroxaban and Edoxaban) or missing the morning dose of a DOAC they take twice per day (Apixaban or Dabigatran) (22). Recent clinical studies have demonstrated that surgical extractions can be completed without interruption of DOAC therapy $(25,26)$. While instances of post-operative haemorrhage following extractions did occur in these studies, there was no significant difference between the incidence of this in the DOAC group compared to the group taking vitamin $\mathrm{K}$ antagonists (e.g. Warfarin Sodium) with levels within the therapeutic range $(25,26)$. Most dentists would be happy to extract teeth for a patient on Warfarin with an INR below 4, so there should be no difference for patients taking a DOAC. In one study there were 2 patients who suffered postoperative haemorrhage in the DOAC group where local measures alone did not control the bleeding (26). In addition to local measures, the patient missed their next dose of the DOAC and the bleeding resolved (26).

In consideration of the risk-benefit ratio for these patients there is an increased risk of bleeding when having any surgical procedure, though it is unlikely to be significant. The bleeding is visible shortly after it starts and is frequently managed by simple local measures. This is inconvenient for the patient but is unlikely to result in any lasting morbidity or mortality. There have been no published instances of significant bleeding following dental treatment for patients taking a DOAC. One paper discussed significant bleeding for a patient who had originally been taking a DOAC but had been switched to Heparin as a bridging agent for the extraction on that occasion (27). The bleeding was managed by local measures (27). The risk of a thrombotic event when stopping a DOAC is not known as there is no clinical research to date. It has been recognised with previous anticoagulants that interrupting therapy can result in a recurrence of a thromboembolic event (28). The risk of an event is very low with the isolated omission of 1 dose, but should it happen the consequences for the patient could be extremely serious. On balance, for a patient who has no other risk factors for post-operative bleeding than a DOAC prescribed at the correct dose corresponding to the patient's kidney function, it would seem hard to justify interrupting DOAC therapy.

\section{Key points}

The key to managing older patients at risk of haemorrhage if an extraction is being considered is to ensure: 
- An accurate risk assessment is undertaken prior to undertaking treatment with particular attention devoted to the medical history, including prescribed medications

- A discussion is undertaken with the patient about the risk of bleeding to enable them to make an informed decision and provide guidance on how to manage this should it occur. However, care must be taken not to alarm the patient to the extent that they then omit doses of medication of their own accord

- Schedule treatment for early in the day

- Ensure local haemostatic measures are used following extractions, such as a haemostatic pack and sutures

\section{Conclusions}

Primary care will continue to become an ever more complex environment in which to work. With the advancements in medical therapies, patients will continue to live longer with increasingly complex medical issues. The medications discussed within this article are not the only medications that affect our surgical branch of healthcare and it is essential that clinicians maintain an up to date knowledge of these drugs and their impacts on oral care.

The developments in antiangiogenic drugs have helped patients with incurable cancer to survive longer. In 2011 it become apparent that unfortunately these drugs also carry a risk of MRONJ (29). These medications include Sunitinib (a tyrosine kinase inhibitor) and Bevacizumab (a vascular endothelial growth factor inhibitor) (29). In 2016 Aflibercept was added to the list, and since then the number these drugs has continued to increase (30). New groups such as mTOR inhibitors have helped to improve progression free survival with breast cancer, but there have been cases of MRONJ for patients on these medications(31). Ibrutinib is an inhibitor of Bruton's tyrosine kinase used for treatment of B-cell lymphoproliferative disorders. It also affects platelet function and results in the patient being at an increased risk of bleeding(32).

While these developments can seem frightening, overall these medications are beneficial for our patients. It highlights the importance of taking a good medical history regularly and communicating with relevant clinicians as required. It is also important to discuss the risks and benefits with patients so they can make informed decisions and know who to contact if something does go wrong. Being able to correctly manage patients taking more common medications such as bisphosphonates, Denosumab, DOACs and dual antiplatelet therapy should be within the remit of primary care clinicians. Many of these patients will, in all likelihood, be most comfortable in a familiar environment with a clinician they have built a relationship with over years. 


\section{References}

1. Information Centre for Health and Social Care Office for National Statistics SSD. Executive summary: Adult Dental Health Survey 2009

https://files.digital.nhs.uk/publicationimport/pub01xxx/pub01086/adul-dent-heal-surv-summ-themexec-2009-rep2.pdf2009 [updated 11/04/2018. Available from:

https://files.digital.nhs.uk/publicationimport/pub01xxx/pub01086/adul-dent-heal-surv-summ-themexec-2009-rep2.pdf.

2. Colella G, Campisi G, Fusco V. American Association of Oral and Maxillofacial Surgeons position paper: Bisphosphonate-Related Osteonecrosis of the Jaws-2009 update: the need to refine the BRONJ definition. J Oral Maxillofac Surg. 2009;67(12):2698-9.

3. Foundation IO. Broken Bones, Broken Lives: A roadmap to solve the fragility fracture crisis in Europe. 2019.

4. Black DM, Cummings SR, Karpf DB, Cauley JA, Thompson DE, Nevitt MC, et al. Randomised trial of effect of alendronate on risk of fracture in women with existing vertebral fractures. Fracture Intervention Trial Research Group. Lancet. 1996;348(9041):1535-41.

5. Papapoulos S, Chapurlat R, Libanati C, Brandi ML, Brown JP, Czerwinski E, et al. Five years of denosumab exposure in women with postmenopausal osteoporosis: results from the first two years of the FREEDOM extension. J Bone Miner Res. 2012;27(3):694-701.

6. Practice FoGD. National study on avascular necrosis of the jaws

including bisphosphonate-related necrosis. 2012.

7. Mavrokokki T, Cheng A, Stein B, Goss A. Nature and frequency of bisphosphonate-associated osteonecrosis of the jaws in Australia. J Oral Maxillofac Surg. 2007;65(3):415-23.

8. Scottish Dental Clinical Effectiveness Programme (SDCEP) achieves NICE accreditation. Evid Based Dent. 2016;17(2):62.

9. Programme SDCE. Oral Health Management of Patients at Risk of Medication-related Osteonecrosis of the Jaw 2017 [Available from: http://www.sdcep.org.uk/wp-

content/uploads/2017/04/SDCEP-Oral-Health-Management-of-Patients-at-Risk-of-MRONJGuidance-full.pdf.

10. Programme SDCE. Oral Health Management of Patients at Risk of Medication-related Osteonecrosis of the Jaw: Guidance in Brief 2017 [Available from: http://www.sdcep.org.uk/wpcontent/uploads/2017/04/SDCEP-Oral-Health-Management-of-Patients-at-Risk-of-MRONJGuidance-in-Brief.pdf.

11. Patntirapong S, Phupunporn P, Vanichtantiphong D, Thanetchaloempong W. Inhibition of macrophage viability by bound and free bisphosphonates. Acta Histochem. 2019;121(4):400-6.

12. Patntirapong $S$, Poolgesorn M. Alteration of macrophage viability, differentiation, and function by bisphosphonates. Oral Dis. 2018;24(7):1294-302.

13. Surgeons AAoOaM. Medication-Related Osteonecrosis of the Jaw - 2014 Update 2014 [Available from:

https://www.aaoms.org/docs/govt affairs/advocacy white papers/mronj position paper.pdf.

14. Lawson MA, Xia Z, Barnett BL, Triffitt JT, Phipps RJ, Dunford JE, et al. Differences between bisphosphonates in binding affinities for hydroxyapatite. J Biomed Mater Res B Appl Biomater. 2010;92(1):149-55.

15. Excellence NIfHaC. Antiplatelet treatment 2018 [Available from:

https://cks.nice.org.uk/antiplatelet-treatment\#!scenario:1.

16. Li L, Zhang W, Yang Y, Zhao L, Zhou X, Zhang J. Dental management of patient with dual antiplatelet therapy: a meta-analysis. Clin Oral Investig. 2019;23(4):1615-23. 
17. Zabojszcz M, Malinowski KP, Janion-Sadowska A, Lillis T, Ziakas A, Slawska A, et al. Safety of dental extractions in patients on dual antiplatelet therapy - a meta-analysis. Postepy Kardiol Interwencyjnej. 2019;15(1):68-73.

18. Lu SY, Lin LH, Hsue SS. Management of dental extractions in patients on warfarin and antiplatelet therapy. J Formos Med Assoc. 2018;117(11):979-86.

19. Babaji P, Rishal Y. Clinical Evaluation of Role of Dual Antiplatelet Therapy on Bleeding after Dental Extraction. Contemp Clin Dent. 2018;9(1):41-4.

20. Lu SY, Tsai CY, Lin LH, Lu SN. Dental extraction without stopping single or dual antiplatelet therapy: results of a retrospective cohort study. Int J Oral Maxillofac Surg. 2016;45(10):1293-8.

21. Doganay O, Atalay B, Karadag E, Aga U, Tugrul M. Bleeding frequency of patients taking ticagrelor, aspirin, clopidogrel, and dual antiplatelet therapy after tooth extraction and minor oral surgery. J Am Dent Assoc. 2018;149(2):132-8.

22. Programme SDCE. Management of Dental Patients Taking Anticoagulants or Antiplatelet Drugs 2015 [Available from: http://www.sdcep.org.uk/wp-content/uploads/2015/09/SDCEPAnticoagulants-Guidance.pdf.

23. Programme SDCE. Management of Dental Patients Taking Anticoagulants or Antiplatelet Drugs: Quick Reference Guide 2015 [Available from: http://www.sdcep.org.uk/wpcontent/uploads/2015/09/SDCEP-Anticoagulants-Quick-Reference-Guide.pdf.

24. Berton F, Costantinides F, Rizzo R, Franco A, Contarin J, Stacchi C, et al. Should we fear direct oral anticoagulants more than vitamin $\mathrm{K}$ antagonists in simple single tooth extraction? A prospective comparative study. Clin Oral Investig. 2019;23(8):3183-92.

25. Mauprivez C, Khonsari RH, Razouk O, Goudot P, Lesclous P, Descroix V. Management of dental extraction in patients undergoing anticoagulant oral direct treatment: a pilot study. Oral Surg Oral Med Oral Pathol Oral Radiol. 2016;122(5):e146-e55.

26. Yoshikawa H, Yoshida M, Yasaka M, Yoshida H, Murasato Y, Fukunaga D, et al. Safety of tooth extraction in patients receiving direct oral anticoagulant treatment versus warfarin: a prospective observation study. Int J Oral Maxillofac Surg. 2019;48(8):1102-8.

27. Ehrhard S, Burkhard JP, Exadaktylos AK, Sauter TC. Severe Enoral Bleeding with a Direct Oral Anticoagulant after Tooth Extraction and Heparin Bridging Treatment. Case Rep Emerg Med. 2019;2019:6208604.

28. Michaels $L$. Recurrence of thromboembolic disease after discontinuing anticoagulant therapy. A study of factors affecting incidence. Br Heart J. 1970;32(3):359-64.

29. Agency MaHpR. Bevacizumab and sunitinib: risk of osteonecrosis of the jaw 2011 [Available from: https://www.gov.uk/drug-safety-update/bevacizumab-and-sunitinib-risk-of-osteonecrosis-ofthe-jaw.

30. Agency MaHpR. Aflibercept (Zaltrap $\mathbf{\nabla}$ ): minimising the risk of osteonecrosis of the jaw 2016 [Available from: https://www.gov.uk/drug-safety-update/aflibercept-zaltrap-minimising-the-risk-ofosteonecrosis-of-the-jaw.

31. Yardley DA, Noguchi S, Pritchard KI, Burris HA, 3rd, Baselga J, Gnant M, et al. Everolimus plus exemestane in postmenopausal patients with $\mathrm{HR}(+)$ breast cancer: BOLERO-2 final progression-free survival analysis. Adv Ther. 2013;30(10):870-84.

32. Shatzel JJ, Olson SR, Tao DL, McCarty OJT, Danilov AV, DeLoughery TG. Ibrutinib-associated bleeding: pathogenesis, management and risk reduction strategies. J Thromb Haemost.

2017;15(5):835-47. 